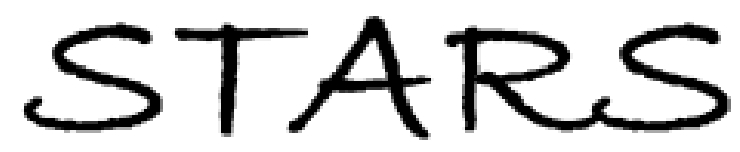

University of Central Florida

STARS

Faculty Bibliography 2010s

Faculty Bibliography

$1-1-2015$

\title{
Gas-focused liquid microjets from a slit
}

\author{
Cheng Li \\ University of Central Florida \\ Kappy Krueger \\ University of Central Florida \\ Weiwei Yang \\ University of Central Florida \\ Hongxu Duan \\ University of Central Florida \\ Weiwei Deng \\ University of Central Florida
}

Find similar works at: https://stars.library.ucf.edu/facultybib2010

University of Central Florida Libraries http://library.ucf.edu

This Article is brought to you for free and open access by the Faculty Bibliography at STARS. It has been accepted for inclusion in Faculty Bibliography 2010 s by an authorized administrator of STARS. For more information, please contactSTARS@ucf.edu.

\section{Recommended Citation}

Li, Cheng; Krueger, Kappy; Yang, Weiwei; Duan, Hongxu; and Deng, Weiwei, "Gas-focused liquid microjets from a slit" (2015). Faculty Bibliography 2010s. 6658.

https://stars.library.ucf.edu/facultybib2010/6658

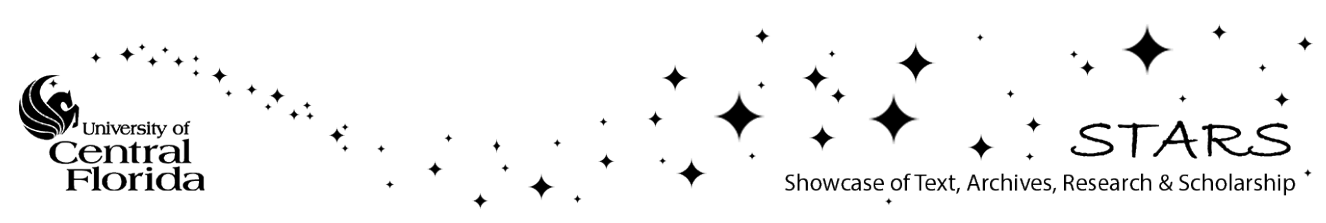




\section{Gas-focused liquid microjets from a slit}

Cheng Li, Kappy Krueger, Weiwei Yang, Hongxu Duan, and Weiwei Deng

Citation: Physics of Fluids 27, 032101 (2015); doi: 10.1063/1.4913721

View online: https://doi.org/10.1063/1.4913721

View Table of Contents: http://aip.scitation.org/toc/phf/27/3

Published by the American Institute of Physics

\section{Articles you may be interested in}

Pinhole formation from liquid metal microdroplets impact on solid surfaces

Applied Physics Letters 108, 041601 (2016); 10.1063/1.4940404

The role of electric charge in microdroplets impacting on conducting surfaces

Physics of Fluids 22, 051703 (2010); 10.1063/1.3431739

Global and local instability of flow focusing: The influence of the geometry

Physics of Fluids 22, 064105 (2010); 10.1063/1.3450321

Breakup of diminutive Rayleigh jets

Physics of Fluids 22, 122003 (2010); 10.1063/1.3524533

Breakup length of forced liquid jets

Physics of Fluids 15, 2469 (2003); 10.1063/1.1593023

Numerical study on droplet generation in axisymmetric flow focusing upon actuation

Physics of Fluids 30, 012111 (2018); 10.1063/1.5009601

\section{PHYSICS TODAY}

WHITEPAPERS

\section{ADVANCED LIGHT CURE ADHESIVES}

Take a closer look at what these environmentally friendly adhesive systems can do
READ NOW

PRESENTED BY

MASTERBOND 


\title{
Gas-focused liquid microjets from a slit
}

\author{
Cheng Li, Kappy Krueger, Weiwei Yang, Hongxu Duan, and Weiwei Deng ${ }^{\text {a) }}$ \\ Department of Mechanical and Aerospace Engineering, University of Central Florida, \\ 4000 Central Florida Blvd., Orlando, Florida 32816, USA
}

(Received 11 January 2015; accepted 9 February 2015; published online 4 March 2015)

\begin{abstract}
We report an experimental study on a new arrangement of flow focusing that uses a slit aperture. The slit can achieve similar flow chocking function as a classic circular aperture to established gas flow pressure drop. Fundamentally, the jetting mechanism and quantitative behavior of slit flow focusing are very similar to the classic flow focusing because of the singularity nature of the jet despite the drastically different aperture shapes. After re-scaling the jet shape, the axial coordinate with the hydraulic diameter of the slit, the experimental data follow closely with the universal jet profile derived in Gañán-Calvo, Ferrera, and Montanero ["Universal size and shape of viscous capillary jets: Application to gas-focused microjets," J. Fluid Mech. 670, 427-438 (2011)] based on circular aperture. Practically, the slit configuration expands the possibility of manipulating the jets. The two thin plates that form the slit aperture can also be used as electrodes to apply electrohydrodynamic excitation to the jet for controlled jet breakup with narrower droplet size distribution. In addition, the modest throughput of a single flow focused jet can be increased by operating a linear array of jets focused and excited by the same slit. ( 2015 AIP Publishing LLC. [http://dx.doi.org/10.1063/1.4913721]
\end{abstract}

\section{INTRODUCTION}

Quasi and strictly monodisperse droplets have found widespread applications such as fabrication of highly uniform micro/nano particles, photonic crystals, and sizing standards. ${ }^{1-5}$ One effective way to generate such uniform droplets is through the breakup of a liquid jet. The ordered jet breakup can be described by Rayleigh's classical linear perturbation theory, ${ }^{6,7}$ which shows that for a liquid jet, perturbations (or excitations) with wavelength longer than the circumference of the jet are unstable and the instability grows exponentially. A fastest growing wavelength of 9.02 times of jet radius exists and often dominates the droplet diameter. This suggests the droplet diameter is about 1.9 times of the jet diameter; therefore, the key to generate small uniform droplets is to form the jet with comparable diameter. Jets can be formed by forcing liquid through a small opening, which becomes increasingly challenging for very fine jets (below $50 \mu \mathrm{m}$ ), primarily due to the higher shear rate caused by the non-slipping solid-liquid interface as the orifice diameter shrinks. The liquid-solid interface can be avoided altogether and replaced with liquid-gas interface by flow focusing ${ }^{8}$ or liquid-liquid interface by double flow focusing. ${ }^{9}$ Fig. 1(a) shows the working principle of the flow focusing device. The liquid is fed through a nozzle with diameter of a few hundred micrometers, and the end of the nozzle is positioned on top of a small circular aperture. A certain gas pressure drop $\Delta \mathrm{P}_{\mathrm{g}}$ is maintained across the aperture. As gas on the nozzle side moves towards the aperture, the pressure loss accelerates the gas to high velocity on the order of $100 \mathrm{~m} / \mathrm{s}$, which is a result of Bernoulli principle. The same pressure gradient also acts on the liquid exiting the nozzle, first deforms the liquid into a conical shape, and eventually accelerates the liquid into a jet at the apex of the cone. This jetting mechanism eliminates the solid-liquid friction and greatly reduced the achievable jet size. Jet diameter as small as

\footnotetext{
a) Author to whom correspondence should be addressed. Electronic mail: weiwei.deng@ucf.edu. Telephone: 407-823-5221. Fax: 407-823-0208.
} 


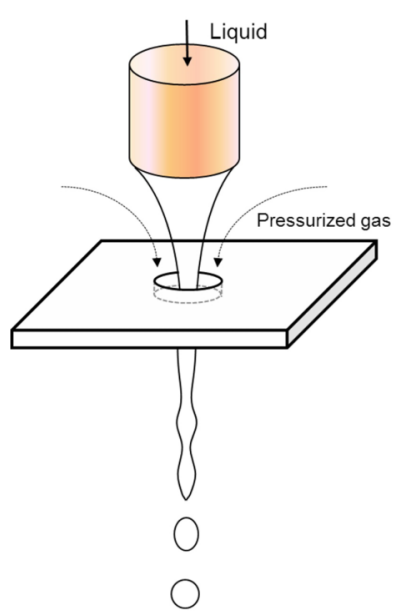

(a)

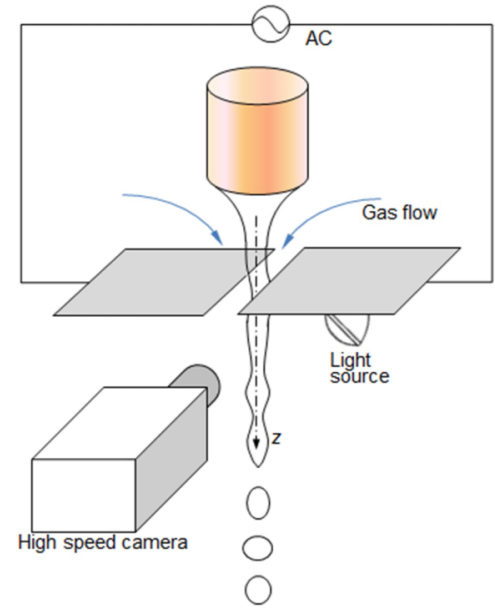

(b)

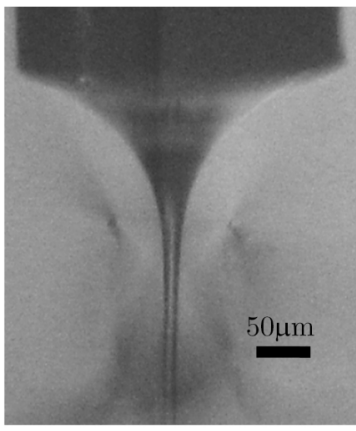

(c)

FIG. 1. (a) Schematic of a typical classic flow focusing device with circular aperture (Ref. 1). (b) Experimental setup of the slit flow focusing device under optional transverse electrohydrodynamic perturbation. (c) Optical photograph of the jet from slit flow focusing. The slit is perpendicular to the image. The liquid is ethanol, flow rate $5 \mathrm{ml} / \mathrm{h}, \Delta \mathrm{Pg}=40 \mathrm{kPa}$.

one micrometer has been reported. ${ }^{10}$ The absence of liquid/solid interface also enables jetting of liquids several hundred times more viscous than water. Such expansion on the viscosity range broadens the selections of liquids, solutions, and suspensions to be processed, such as polymer solutions and suspensions with high concentrations of solid particle loadings.

Flow focusing of liquid in gas phase medium is essentially a fluid extrusion mechanism that uses the pneumatic pressure as the energy source. Gañan-Calvo et al. have formulated a unified theory ${ }^{11}$ that can treat other different energy sources such as electric potential drop, gravity, and pneumatic pressure drop. The model can also describe both the inviscid jetting and highly viscous jetting cases, and the latter has a jet radius that is inverse $1 / 4$ power of the axial distance away from where the liquid is discharged.

All reported previous follow focusing works use circular aperture as the "choking" mechanism for maintaining the gas pressure drop, and we refer it as classic flow focusing. The pressure drop may also be established by other type of small openings, such as a slit. In this paper, we study experimentally the slit flow focusing and show that liquid meniscus suspended on a nozzle can indeed be focused by a slit opening. The slit flow focusing may simplify the assembly and operation because only one dimensional alignment (instead of two dimensional for the circular apertures) is required between the slit and the nozzle. Slit flow focusing is also readily scalable by aligning multiple nozzles with the same slit. The scalability is important because a single flow focusing source can only handle liquid flow rates of $\sim 10 \mathrm{ml} / \mathrm{h}$, which may be too low to be a viable atomization approach for industry such as manufacturing monodisperse particles. Further, the two thin plates that form the slit can serve as another function of electrohydrodynamic (EHD) exciter, which may provide transverse electrohydrodynamic perturbation ${ }^{12}$ to improve the monodispersity of the droplet size.

We shall restrict the scope of the present work in the "intact jet region" 3,4 and the quantification of the jet profile as a function of liquid viscosities, flow rates, and pressure drops. The phenomena of jet instabilities ${ }^{13,14}$ (such as varicose or whipping) of the slit focusing jet are not the focus of this work (with the only exception being that we demonstrate the feasibility of using electrohydrodynamic excitation to prescribe a dominant breakup frequency for improved droplet size distribution in Sec. III D).

\section{EXPERIMENTAL}

Fig. 1(b) illustrates the slit flow focusing experimental setup. The major difference between the slit flow focusing to the classic flow focusing ${ }^{8}$ device is that the circular hole is replaced by a slit 
TABLE I. Properties of liquids used in this work.

\begin{tabular}{lccr}
\hline \hline Liquid & Viscosity (mPa s) & Surface tension $(\mathrm{mN} / \mathrm{m})$ & Density $\left(\mathrm{kg} / \mathrm{m}^{3}\right)$ \\
\hline Water & 1.0 & 73.0 & 1000 \\
Ethanol & 1.2 & 22.2 & 790 \\
EG-10 (68 wt. \% ethanol, 32 wt. \% glycerol) & 12.0 & 23.5 & 946 \\
EG-100 (36 wt. \% ethanol, 64 wt. \% glycerol) & 120 & 25.1 & 1020 \\
\hline \hline
\end{tabular}

aperture, which was made in-house by putting two thin plates (such as razor blades or glass slides) together. The slit opening $w$ is fully adjustable from 50 to $200 \mu \mathrm{m}$. The two blades are electrically insulated from each other for the purpose of applying EHD perturbation, which will be discussed in Sec. III D. The liquids tested include ethanol, deionized water, and mixture of ethanol and glycerol to formulate a selection of liquids with different viscosities. The liquid property is taken from the literature ${ }^{15}$ and is summarized in Table I. Particularly, the mixtures of (68 wt. \% ethanol $+32 \mathrm{wt} . \%$ glycerol) and (36 wt. \% ethanol $+64 \mathrm{wt} . \%$ glycerol) are termed as EG-10 and EG-100 because their viscosities are 10 times and 100 times of that of pure ethanol, respectively.

At suitable operating parameters, the gas flow will focus the liquid meniscus into a cone-jet. The phenomena were recorded with a high speed camera (Phantom v12.1) or a digital single-lens reflex camera (Canon 650D) coupled with a microscope lens and light -emitting diode (LED) flash (1 $\mu$ s pulse). Fig. 1(c) is the optical photograph of the slit focusing showing the two thin glass slides (150 $\mu$ m-thick) bracketing the cone-jet. The jet diameter is measured from the image at the location as far away from the nozzle as possible, given the condition that the measurement point is still in sharp focus with good contrast. The imaging system can resolve the jet diameter with an uncertainty of $\pm 1 \mu \mathrm{m}$.

\section{RESULTS AND DISCUSSIONS}

\section{A. Operating conditions and general behavior of slit flow focusing}

To estimate the initial operating conditions (i.e., geometrical arrangement, liquid flow rate, and gas pressure drop), we drew insights into previous experimental work ${ }^{1,13-16}$ as well as numerical simulations ${ }^{17,18}$ of closely related follow focusing with circular holes.

For the classic flow focusing with circular holes, the Weber number should fall in a certain range to establish stable flow focused jets. The Weber number is usually defined as $W e=\rho_{j} v_{j}^{2} d / \gamma$, where $\rho_{j}$ is the liquid density, $v_{j}$ is jet velocity, $d$ is jet diameter, and $\gamma$ is the liquid-gas interfacial tension. Because $\Delta \mathrm{P}_{\mathrm{g}} \sim \rho_{j} v_{j}{ }^{2}$, Weber number needs to be sufficiently large to ensure sufficient pressure drop to overcome the liquid surface tension and deform the liquid meniscus into conical shape. Weber number also cannot be too large, otherwise non-symmetric perturbation will grow and the jet will break up chaotically, ${ }^{1,16}$ which defeats the purpose of achieving high level of monodispersity of the generated droplets. Gañan-Calvo and Barrero ${ }^{8}$ found that $1<W e<40$ is appropriate for flow focused jets with circular aperture configuration. We argue that this desired range of Weber number is also approximately applicable to the slit flow focusing. This is because the focusing of the liquid flow relies on the gas flow pressure drop, which is established and maintained by the flow restriction or "choking." Both round holes and slit openings can achieve similar effect of flow restriction. On the other hand, the jet radius is small compared to the opening of round hole or slit; therefore, the exact geometry choice (circular hole or slit) to restrict the gas flow is not critical.

The geometry effects on classic flow focusing have been analyzed by Vega et al. ${ }^{14}$ where the ratio between nozzle height and aperture size affects operation regions. In slit flow focusing configuration, the understanding of gas flow field can shed light on the device design. To that end, we solve the gas flow field analytically by neglecting the effect of nozzle and liquid meniscus on the gas flow. We further assume the gas flow is two dimensional, laminar, and incompressible. The incompressible flow is a reasonable approximation because the typical pressure drop $\Delta \mathrm{P}_{\mathrm{g}}$ is less than $10 \mathrm{kPa}$, or $10 \%$ of atmosphere pressure. Thus, the flow through the slit becomes a classic potential flow problem which can be solved analytically by conformal mapping. Although this flow 

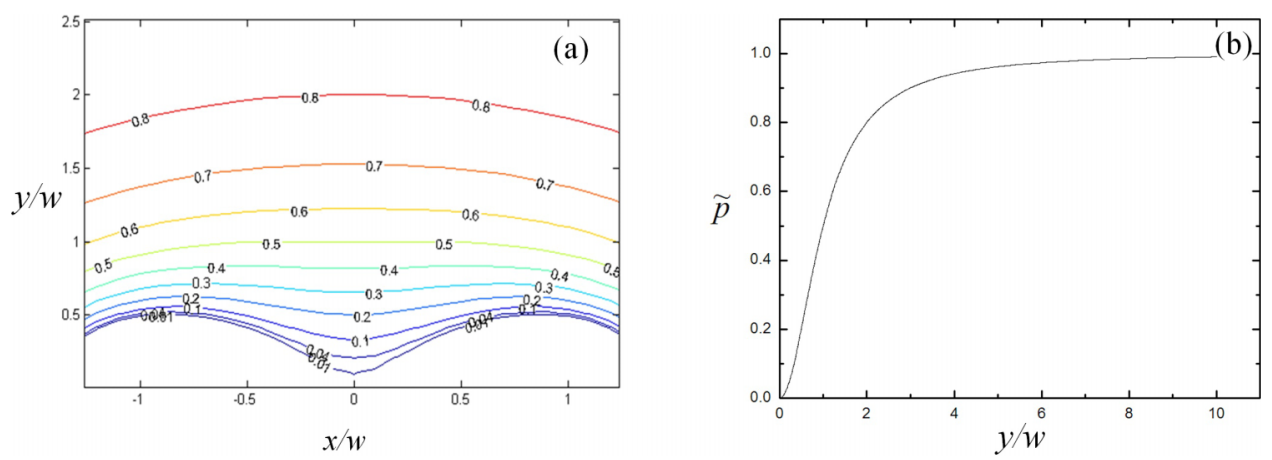

FIG. 2. (a) Dimensionless equal pressure lines inside the chamber. (b) Dimensionless pressure along $y$ direction, with $y=0$ is at the same plane of the slit aperture.

field solution is approximate due to the assumptions we made, it may still provide guidelines on device design and operation. Fig. 2(b) shows the pressure field inferred from the flow field solution. The pressure is normalized by $\rho_{g} Q_{g}{ }^{2} / 2 w^{2} \pi^{2}$, where $\rho_{g}$ is the gas density, $Q_{g}$ is the gas flow rate, and $w$ is the slit opening width. Fig. 2(b) suggests that the pressure drop is half of the maximum value when the nozzle is positioned at $y / w=1$. The pressure drop is approximately linearly dependent on $y$ till $y / w \approx 2$. This result suggests that in order to harvest the majority of the pressure drop, the nozzle should be placed above the gap center at a distance of $y / w \sim 2$.

The analysis above suggests that the asymptotic behavior of the transition from liquid meniscus to jet will be completed within an axial distance of $\sim 2 w$. In other words, although the base diameter of the nozzle or the distance of the nozzle tip to the slit opening can be larger than $2 w$, the region with substantial meniscus diameter draw-down is about twice of the slit opening. Fig. 3 shows the images of several geometrical arrangements of the nozzle to the slit $(w=50 \mu \mathrm{m})$ formed by two glass slides. In the top row in Fig. 3, the cone-jet structure can be maintained for a wide range of vertical distance between the nozzle and the slit ( $y=$ from $300 \mu \mathrm{m}$ to $80 \mu \mathrm{m})$. The bottom row of Fig. 3 shows various scenarios where the nozzle is deliberately misaligned with the slit. For a lateral
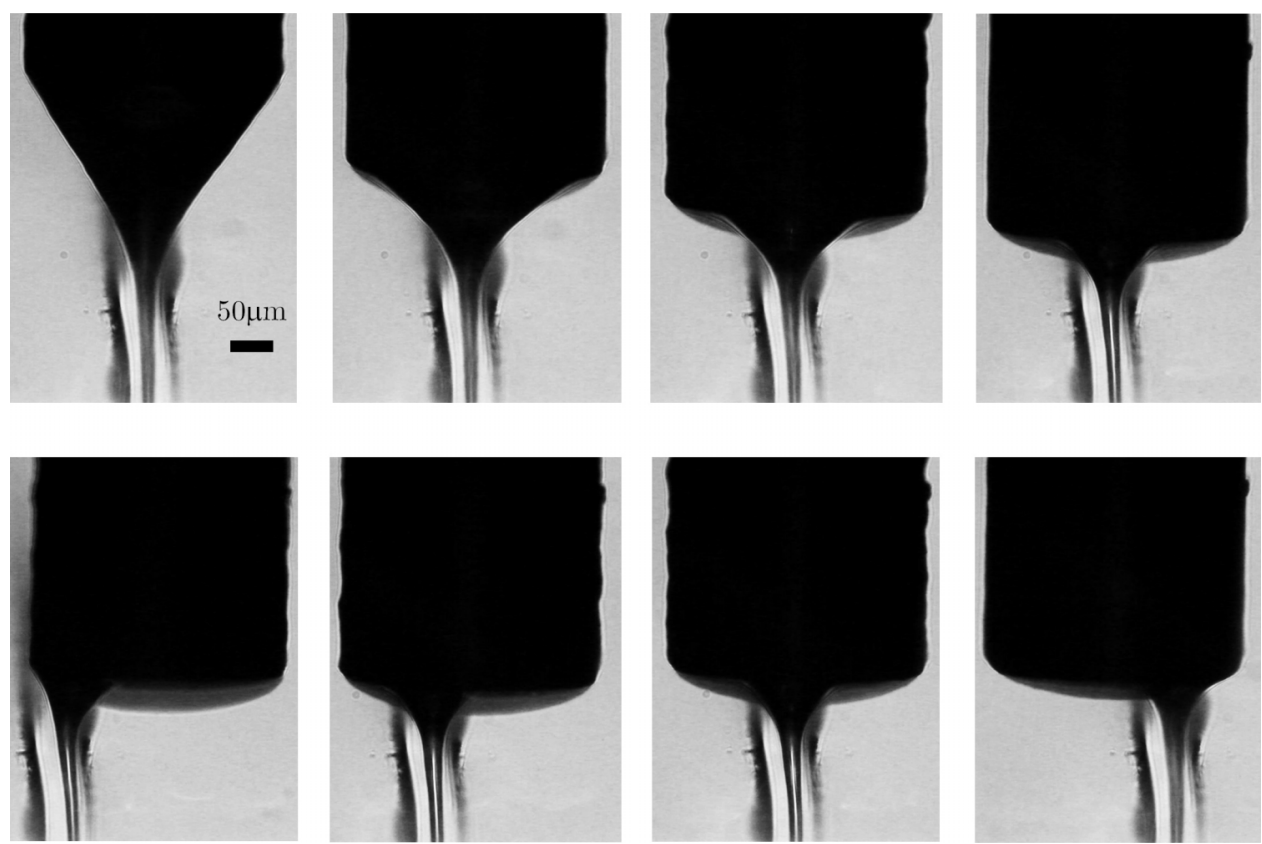

FIG. 3. Jet profile as the lens optical axis is parallel to the slit. The jet appears to be very robust as the jet shifts left and right or up and down. 


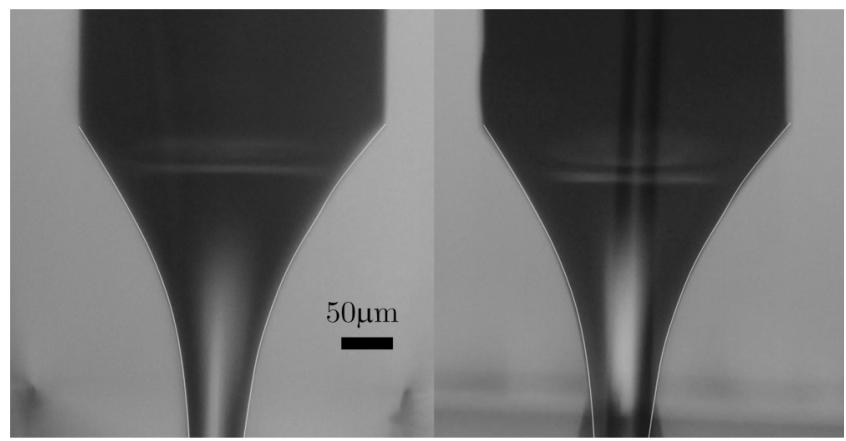

(a)

(b)

FIG. 4. Liquid cone profile taken from two orthogonal viewing directions. (a) Slit is perpendicular to the image. (b) Slit is parallel to the image.

change of as large as one time of the nozzle diameter (300 $\mu \mathrm{m}$ in this case), the stable cone-jet firmly anchors on the nozzle. The jet diameter is not affected by the relative position of the nozzle to the slit. This is consistent with the asymptotic jetting behavior with a characteristic length of $\sim 2 w$.

Because the gas flow field is not axisymmetric, it may cause the cross section shape of the cone and jet to be non-circular. To examine this possibility, we photographed the same jetting process from two orthogonal viewing directions. Fig. 4(a) was taken as the slit is perpendicular to the image, while in Fig. 4(b), the slit was parallel to the image. The edge of the liquid jet in Fig. 4(a) is marked in white curves, and the same white curves are super-imposed in Fig. 4(b) to allow a direct comparison of the cone/jet profiles from two orthogonal directions. The two jet profiles appear to be identical within the resolving limit of the imaging system $(\sim 1 \mu \mathrm{m})$. Therefore, we conclude that the effect of the slit on the roundness of the jet is negligible, again as a consequence of singularity of the cone and jet.

\section{B. Jet radius}

The nearly axisymmetric nature of the jet (despite using the slit configuration) suggests that the detailed outer gas flow field has minor effect on jetting dynamics, and the outcome should be governed by the same set of momentum equation as the flow focusing through circular holes ${ }^{1}$ with the jet radius determined by the same scaling law

$$
R \equiv\left(\frac{\rho_{j}}{2 \pi^{2}}\right)^{1 / 4}\left(\Delta P_{g}\right)^{-1 / 4} Q^{1 / 2},
$$

where $Q$ is the liquid flow rate.

Figs. 5 and 6 show the experimental measurement from the four types of liquids with different viscosity and surface tension as listed in Table I. The triangle in Fig. 5 denotes the slope of $-1 / 4$ in the Log-Log plot. For fixed flow rates (Fig. 5), all data sets of the jet radius versus the pressure approximately follow the $-1 / 4$ slope, which is consistent with Eq. (1). Similarly, in the jet radius versus flow rate plot (Fig. 6), all data sets approximately follow the slope of $1 / 2$ indicated by the triangle in Fig. 6. Figs. 5 and 6 support the notion that Eq. (1), despite being derived from circular aperture flow focusing, applies well to the slit flow focusing.

The dimensionless radius $R_{j} / R$ (measured value) divided by the estimate from Eq. (1) versus the Weber number is plotted in Fig. 7 for the four liquids. The Weber number is re-defined as in Gañán-Calvo et al. ${ }^{11}$

$$
W e=\left(\frac{8}{\pi^{2}}\right)^{1 / 4}\left(\frac{\Delta P_{g}^{3} \rho_{j} Q^{2}}{\sigma^{4}}\right)^{1 / 4} .
$$

Noticeably, $R_{j} / R$ is very close to unity for all the cases measured and analyzed. This indicates that Eq. (1) derived from classic flow focusing is an excellent model for predicting the radius of 


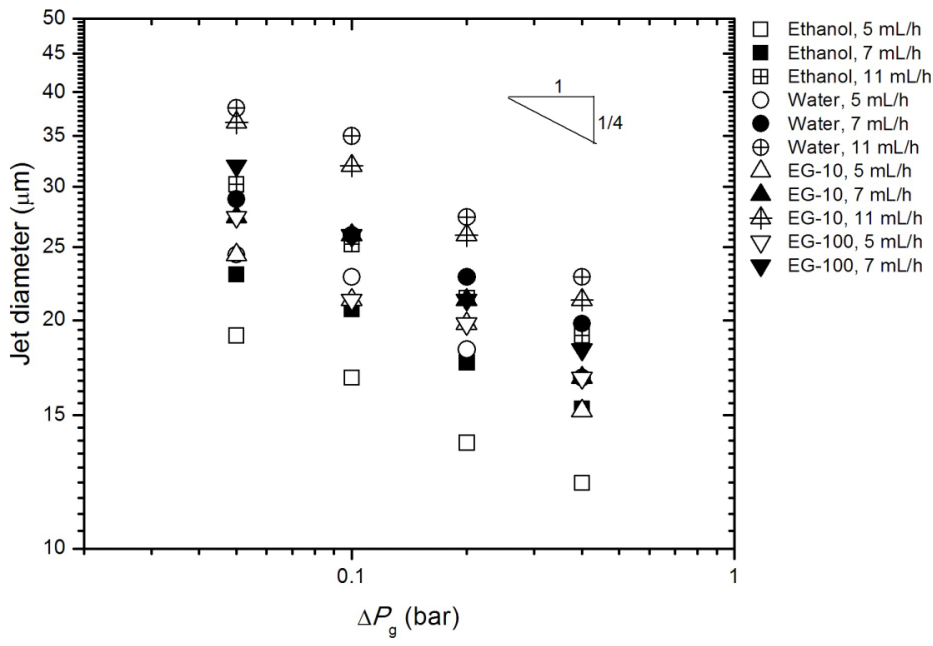

FIG. 5. Jet radius of various liquids at different pressures.

the jet focused by a slit. In addition, for all cases shown, We is always greater than 1 . This is expected as the inertia has to overcome the surface tension for jetting to occur, regardless of the focusing geometry (round hole or slit). This observation is consistent with the classic flow focusing reported previously (Gañán-Calvo et al. ${ }^{11}$ ) in which the We $\sim 1$ is considered as a global stability limit corresponding to the transition of jetting to dripping.

\section{Universal jet profile}

Next, we investigate whether the universal scaling laws proposed in Gañán-Calvo et al. ${ }^{11}$ based on the slender approximation can be used to describe jets from slit flow focusing. To that end, we follow the notations of Gañán-Calvo et al. ${ }^{11}$ and define the dimensionless jet radius $\xi$ and the axial coordinate $\eta$

$$
\xi=R_{\mu} / R, \eta=z / D_{s},
$$

where $D_{s}$ is the characteristic length of the slit, $z$ is the axial location, and $R_{\mu}$ is the characteristic jet radius

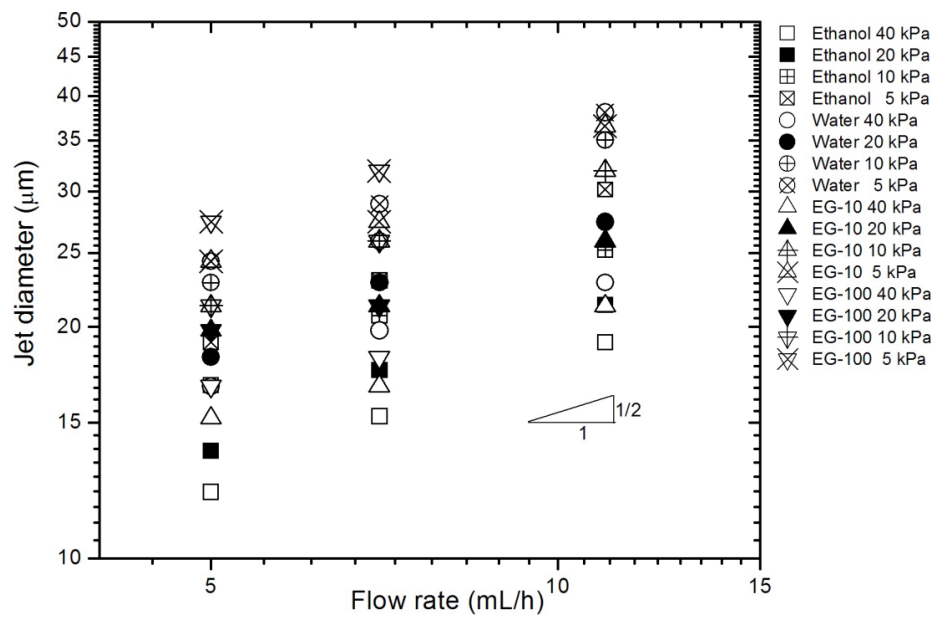

FIG. 6. Jet radius of various liquids at different flow rates. 


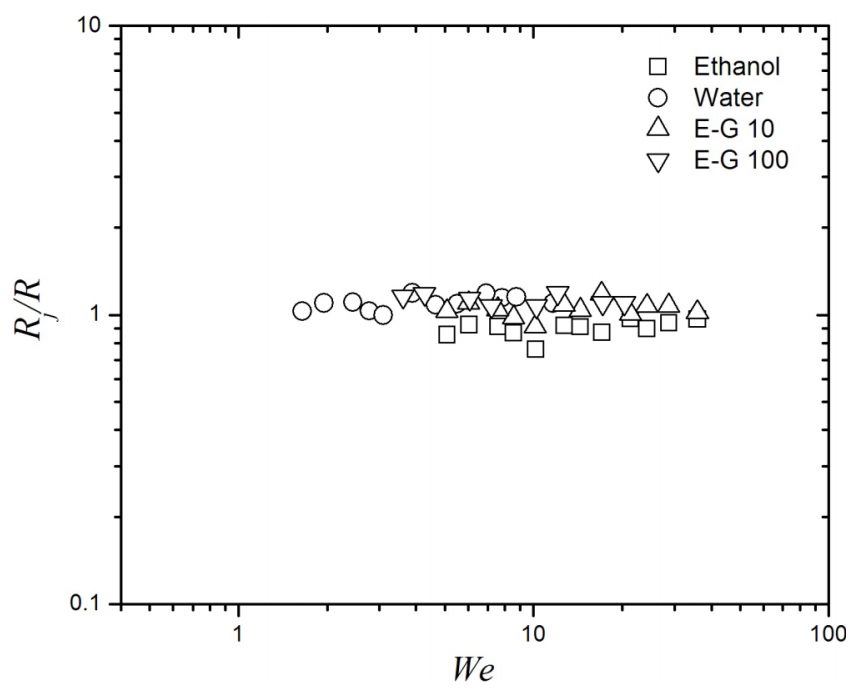

FIG. 7. Dimensionless jet radius as a function of Weber number.

$$
R_{\mu}=\left(\frac{\mu_{l}^{2}}{\rho_{j} \Delta P_{g}}\right)^{1 / 2} .
$$

Here, $\mu_{l}$ is the liquid viscosity. The re-scaled jet velocity and axial coordinate are

$$
\begin{gathered}
v \equiv 2\left(6 \sqrt{2} R_{\mu} / D_{s}\right)^{-1 / 3} \xi^{-2}, \\
x \equiv 2\left(6 \sqrt{2} R_{\mu} / D_{s}\right)^{-2 / 3} \eta .
\end{gathered}
$$

These re-scaled variables together with the slender assumption allow Gañan-Calvo et al. ${ }^{11}$ to derive a self-similar differential equation of the jet profile in terms of $v$ and $x$ with a remarkably accurate approximate solution (Eq. (2.12) in Gañán-Calvo et al. $)^{11}$

$$
v \equiv\left(\frac{1}{(2 x)^{1 / 2}}+\frac{2}{x^{2}}\right)^{-1} .
$$

In classic flow focusing, the characteristic length of the opening is simply the diameter of the circular aperture. For slit flow focusing, the choice of slit width as $D_{s}$ is not suitable as it does not take into account of the long end of the slit. In fact, the hydraulic diameter of a slit (equivalent to twice of the slit width) is a more appropriate choice for the characteristic diameter. Fig. 8 shows comparison between the experimental data (open circles) and Eq. (7) (solid line) in terms of the re-scaled variables based on $D_{s}=2 w$. Fig. 8 illustrates that the experimental results agree well with Eq. (7), which is based on the circular aperture flow focusing.

\section{The advantages and limitations of slit flow focusing}

In general, the operation of flow focusing (both round and slit apertures) is very robust in terms of tolerating the misalignment between the nozzle and aperture, as Fig. 3 suggests. In addition, slit flow focusing has its own unique characteristics brought by the slit configuration.

First of all, slit flow focusing is readily scalable to a linear array of nozzles because the same slit can apply to multiple jets with relative alignment ease. The scale-up or multiplexing of the flow focused jets is highly desirable for boosting the throughput of liquid being processed, because the single flow focused jet can only deliver low flow rate of $\sim 10 \mathrm{ml} / \mathrm{h}$. Fig. 9 demonstrates this concept of multiplexed slit flow focusing. A four-nozzle linear array was machined using a precision drilling and milling process out of aluminum. ${ }^{19}$ The nozzles (Fig. 9(a)) have equal spacing of $500 \mu \mathrm{m}$, outer diameter (OD) of $200 \mu \mathrm{m}$, and inner diameter (ID) of $100 \mu \mathrm{m}$. The nozzle array is aligned with the slit, which is $\sim 1 \mathrm{~mm}$ away from the nozzles. The slit is formed by putting two razor blades near 


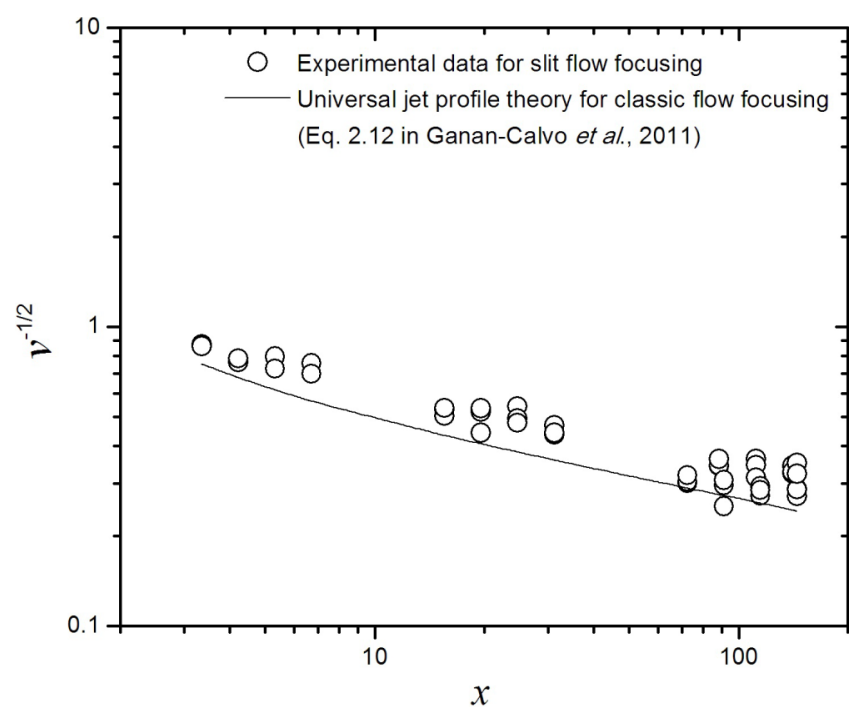

FIG. 8. Universal jet shape $v^{-1 / 2}$ as a function of the re-scaled axial coordinate $x$.

each other. The blade plane is perpendicular to the jets and the blade edges are parallel to the jet array. The device generates four flow focused jets simultaneously from the same slit (Fig. 9(b)).

Interestingly, the blades used to form the slit aperture can also function as electrodes that apply transverse electrohydrodynamic excitation to the jets. ${ }^{12}$ The excitation or perturbation can be in mechanical, acoustic, or electrohydrodynamic forms. ${ }^{2,3,20}$ When properly tuned, the excitation may trigger certain instability at the fastest growth rates of varicose mode of the jet instability. As a result, the excitation may convert the random natural breakup of jets into highly ordered and reproducible jet breakup at a prescribed frequency. With properly applied electrohydrodynamic excitation, the droplet size monodispersity can be noticeably improved. Fig. 9(c) shows the result after we applied a sinusoidal alternating current (AC) signal source to the blades with $V_{\mathrm{p}-\mathrm{p}}$ (peak-to-peak voltage) of $400 \mathrm{~V}$ and frequency of $5 \mathrm{kHz}$. The jet Weber number here is from 2 to 6 as found by Si et al. ${ }^{16}$ for it to operate under axisymmetric mode. After the electrohydrodynamic excitation is applied, the jet breakup location moved closer to the nozzles by $\sim 1 \mathrm{~mm}$. Note that the microdrilling did not produce perfectly identical inner diameter of the four nozzles, and as a result, the right most

(a)
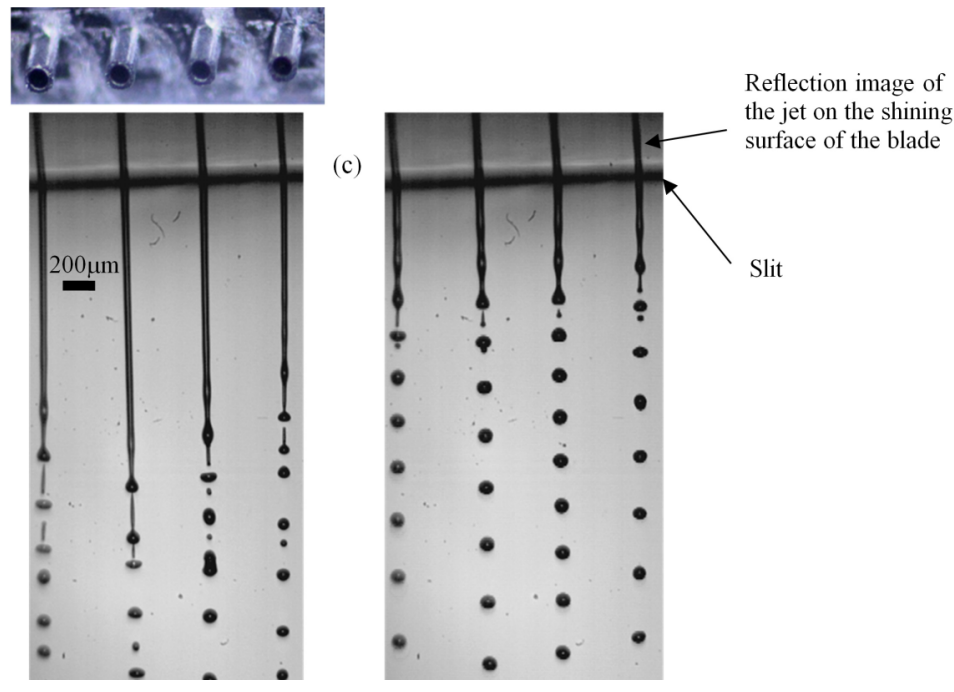

FIG. 9. Scale up of slit flow focusing. (a) The manifold of four aluminum nozzles (nozzle spacing: $500 \mu \mathrm{m}$; nozzle $\mathrm{OD}=200 \mu \mathrm{m}, \mathrm{ID}=100 \mu \mathrm{m}$ ). (b) Jet breakup before EHD excitation. (c) Jet breakup after EHD excitation. 
nozzle passes less flow rate and forms a thinner jet than the rest. Nevertheless, the breakup of all four jets is very regular and repeatable, as evidenced by the equal spacing of droplets from four jets. Under the electrohydrodynamic excitation, one droplet is generated during each half AC cycle for each jet. The space between the adjacent droplets is the product of half AC period and the jet velocity. A more thorough discussion on the effect of transverse electrohydrodynamic excitation on the micro-jet can be found in Yang et al. ${ }^{12}$

One potential limitation of the slit flow focusing is the higher gas flow rate required. To achieve the flow focusing, the same gas pressure drop $\Delta \mathrm{P}_{\mathrm{g}}$ needs to be established for the circular or slit aperture. This suggests the comparable gas exit velocity needs to be maintained, and the gas flow rate is proportional to the area of the aperture. A slit aperture demands higher gas flow rate as the slit may be several times larger than the circular aperture. However, it is worth mentioning that this limitation diminishes for multiplexed jets, especially when packed closely together. Take Fig. 9, for example, the $500 \mu \mathrm{m}$ spacing between two jets and the $\sim 200 \mu \mathrm{m}$ slit opening width translate to an average aperture area of $\sim 0.1 \mathrm{~mm}^{2}$ per jet, and this area is comparable with the area of a circular aperture of $\sim 300 \mu \mathrm{m}$ in diameter.

\section{CONCLUSION}

This paper reports a new approach for generating microjets based on slit flow focusing. The slit can achieve similar flow chocking function as a circular hole ${ }^{1}$ to established gas flow pressure drop which is used in all previous flow focusing devices. The same jet diameter scaling law ${ }^{1}$ derived from classic flow focusing also applies to slit flow focusing. Moreover, after re-scaling the jet shape, the axial coordinate with the hydraulic diameter of the slit, the experimental data follow closely with the universal jet profile derived in Gañán-Calvo and Montanero ${ }^{11}$ based on circular aperture. Fundamentally, the jetting mechanism and quantitative behavior are insensitive to the shape of the focusing aperture because of the singularity nature of the jet. Practically, the slit configuration expands the possibility of manipulating the jets. For example, the two thin plates can serve dual function of forming the slit aperture and applying electrohydrodynamic excitation to the jet for controlled jet breakup with narrower droplet size distribution. In addition, the modest throughput of a single flow focused jet can be increased by operating a linear array of jets focused and excited by the same slit.

\section{ACKNOWLEDGMENTS}

We thank the National Science Foundation (Grant Nos. CMMI 1335295, CMMI 1301099, and CMMI 1454406) for financial support.

${ }^{1}$ A. M. Gañán-Calvo, "Generation of steady liquid microthreads and micron-sized monodisperse sprays in gas streams," Phys. Rev. Lett. 80, 285 (1998).

${ }^{2}$ H. González and F. J. García, "The measurement of growth rates in capillary jets,” J. Fluid Mech. 619, 179 (2009).

${ }^{3}$ J. Eggers, "Nonlinear dynamics and breakup of free-surface flows," Rev. Mod. Phys. 69, 865 (1997).

${ }^{4}$ J. Eggers and E. Villermaux, "Physics of liquid jets," Rep. Prog. Phys. 71, 036601 (2008).

${ }^{5}$ R. Vehring, W. R. Foss, and D. Lechuga-Ballesteros, "Particle formation in spray drying," J. Aerosol. Sci. 38, 728 (2007)

${ }^{6}$ J. W. S. Lord Rayleigh, "On the instability of jets," Proc. London Math. Soc. 10, 4 (1879).

${ }^{7}$ S. Chandrasekhar, Hydrodynamic and Hydromagnetic Stability (Courier Dover Publications, 1961).

${ }^{8}$ A. M. Gañán-Calvo and A. Barrero, "A novel pneumatic technique to generate steady capillary microjets," J. Aerosol. Sci. 30, 117 (1999).

${ }^{9}$ A. M. Gañán-Calvo, R. González-Prieto, P. Riesco-Chueca, M. A. Herrada, and M. Flores-Mosquera, "Focusing capillary jets close to the continuum limit," Nat. Phys. 3, 737 (2007).

${ }^{10}$ A. J. Acero, N. Rebollo-Muñoz, J. M. Montanero, A. M. Gañán-Calvo, and E. J. Vega, "A new flow focusing technique to produce very thin jets," J. Micromech. Microeng. 23, 065009 (2013).

11 A. M. Gañán-Calvo, C. Ferrera, and J. M. Montanero, "Universal size and shape of viscous capillary jets: Application to gas-focused microjets," J. Fluid Mech. 670, 427-438 (2011).

12 W. Yang, H. Duan, C. Li, and W. Deng, "Crossover of varicose and whipping instabilities in electrified microjets," Phys. Rev. Lett. 112, 054501 (2014).

${ }^{13}$ J. M. Gordillo, M. Pérez-Saborid, and A. M. Gañán-Calvo, "Linear stability of co-flowing liquid-gas jets," J. Fluid Mech. 448, 23-51 (2001).

${ }^{14}$ E. J. Vega, J. M. Montanero, M. A. Herrada, and A. M. Gañán-Calvo, "Global and local instability of flow focusing: The influence of the geometry," Phys. Fluids 22, 064105 (2010). 
${ }^{15}$ A. S. Alkindi, Y. M. Al-Wahaibi, and A. H. Muggeridge, "Physical properties (density, excess molar volume, viscosity, surface tension, and refractive index) of ethanol + glycerol," J. Chem. Eng. Data 53, 2793-2976 (2008).

${ }^{16}$ T. Si, F. Li, X.-Y. Yin, and X.-Z. Yin, "Modes in flow focusing and instability of coaxial liquid-gas jets," J. Fluid Mech. 629, 1 (2009).

${ }^{17}$ R. Suryo and O. A. Basaran, "Tip streaming from a liquid drop forming from a tube in a co-flowing outer fluid," Phys. Fluids 18, $082102(2006)$

${ }^{18}$ M. A. Herrada, A. M. Gañán-Calvo, A. Ojeda-Monge, B. Bluth, and P. Riesco-Chueca, "Liquid flow focused by a gas: Jetting, dripping, and recirculation," Phys. Rev. E 78, 036323 (2008).

${ }^{19}$ B. Lojewski, W. Yang, H. Duan, C. Xu, and W. Deng, "Design, fabrication, and characterization of linear multiplexed electrospray atomizers micro-machined from metal and polymers," Aerosol. Sci. Technol. 47, 146 (2013).

${ }^{20}$ R. N. Berglund and B. Y. Liu, "Generation of monodisperse aerosol standards," Environ. Sci. Technol. 7(2), 147-153 (1973). 